\title{
Determination of plastic properties of metals by instrumented indentation using a stochastic optimization algorithm
}

\author{
I. Peyrot \\ Laboratory for Mechanics of Materials and Nanostructures, EMPA-Swiss Federal Laboratories \\ for Materials Testing and Research, 3602 Thun, Switzerland \\ P-O. Bouchard \\ Mines ParisTech, CEMEF, Centre de Mise en Forme des Matériaux, UMR CNRS 7635, 06904 \\ Sophia-Antipolis Cedex, France \\ R. Ghisleni and J. Michler ${ }^{\text {a) }}$ \\ Laboratory for Mechanics of Materials and Nanostructures, EMPA-Swiss Federal Laboratories \\ for Materials Testing and Research, 3602 Thun, Switzerland
}

(Received 31 July 2008; accepted 28 October 2008)

\begin{abstract}
A novel optimization approach, capable of extracting the mechanical properties of an elasto-plastic material from indentation data, is proposed. Theoretical verification is performed on two simulated configurations. The first is based on the analysis of the load-displacement data and the topography of the residual imprint of a single conical indenter. The second is based on the load-displacement data obtained from two conical indenters with different semi-angles. In both cases, a semi-analytical approach [e.g., Dao et al., Acta Mater. 49, 3899 (2001) and Bucaille et al., Acta Mater. 51, 1663 (2003)] is used to estimate Young's modulus, yield stress, and strain hardening coefficient from the load-displacement data. An inverse finite element model, based on a commercial solver and a newly developed optimization algorithm based on a robust stochastic methodology, uses these approximate values as starting values to identify parameters with high accuracy. Both configurations use multiple data sets to extract the elastic-plastic material properties; this allows the mechanical properties of materials to be determined in a robust way.
\end{abstract}

\section{INTRODUCTION}

In recent years, instrumented indentation has developed into an effective, non-destructive method for evaluating the mechanical properties of metallic materials at the nanoscale. ${ }^{1-3}$ The standard test for determining the mechanical properties is the tensile test; however, this requires more complex testing equipment and the preparation of tensile test samples. With indentation, the Young's modulus is estimated by analyzing the slope of the unloading part of the load $(L)$ and penetration depth ( $h$ ) curve (hereafter called $L-h$ curve) and the indenter contact area. ${ }^{4,5}$ The determination of the plastic properties from the loading part of the $L-h$ curve of metals was first obtained in the form of empirical relationships, which directly correlated hardness with yield stress and tensile strength. ${ }^{5,6}$ The ideal conversion of the $L-h$ data to the stress-strain data (hereafter called $\sigma-\varepsilon$ curve) is more challenging since there is not a direct relationship between the $L-h$ curve and the $\sigma-\varepsilon$ curve. $^{7,8}$ This problem became the goal of several scientists, who recently

\footnotetext{
a) Address all correspondence to this author.

e-mail: Johann.Michler@empa.ch

DOI: 10.1557/JMR.2009.0118
}

advanced different models that estimate $\sigma-\varepsilon$ curves from $L-h$ curves. $^{8-14}$ The investigation of new and improved models allowing the determination of the plastic properties of metals is still widely studied today. ${ }^{15-20}$ Reviews of the existing models have been published by Capehart and Cheng ${ }^{21}$ and by Gouldstone et al. ${ }^{22}$

To increase the robustness of the semi-analytical approach presented here, multiple input data sets have been coupled, ${ }^{10,11}$ and recently in-depth sensitivity analysis has been performed to determine the sensitivity and robustness of the results. ${ }^{23-24}$

The inverse method developed consists of fitting a constitutive law to a set of input data (see Fig. 1). Simulated data were used as target data throughout this investigation to perform a theoretical verification of the optimization approach put forward. Experimental verification, using experimental data as input data, will follow. It leads to the formulation of a general multiobjective optimization problem, which consists of a number of objectives to be optimized simultaneously and is associated with a number of inequality and equality constraints. ${ }^{25}$ Optimization algorithms can be classified in three main categories: deterministic algorithms (gradient-based approaches), stochastic algorithms, and 


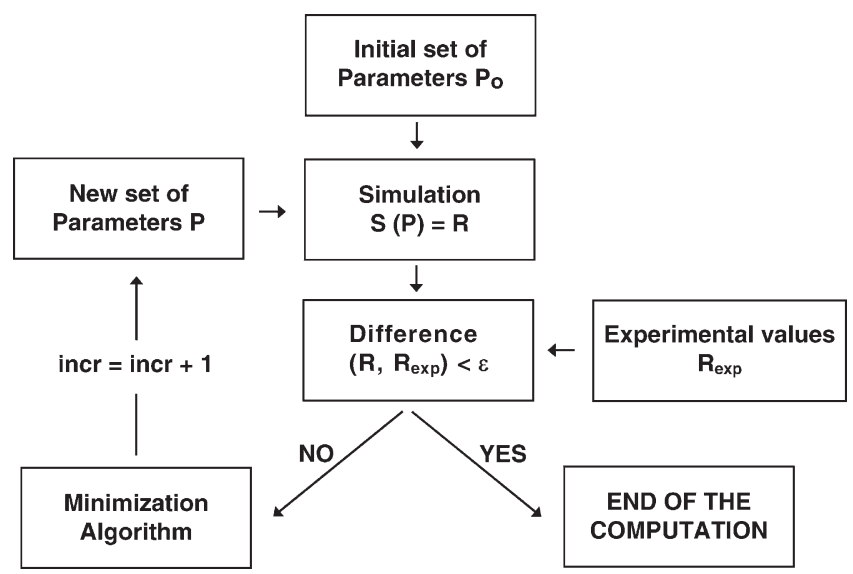

FIG. 1. Inverse method illustrating the different steps in the numerical optimization procedure.

hybrid formulations. Deterministic approaches ${ }^{26}$ are generally based on robust and efficient methods that require the computation of the gradient of the objective function. The solution is generally found in few iterations but may depend on the starting set of parameters, and these algorithms may also be trapped in local optima, especially for highly nonlinear problems. The program SiDoLo, ${ }^{27}$ based on the gradient-based approach, was used to identify the elastic plastic parameters of the indented materials. To avoid the problem of a local minimum, Bocciarelli and Maier $^{28}$ as well as Stauss $^{29}$ have obtained good results by coupling the simulated $L-h$ curve and the simulated profile curve (topography of the indented material after unloading) to the experimental ones. However, they were confronted by a large dependence of the solution on the initial values. Stochastic methods are search-based algorithms in which no evaluation of the gradient of the objective function is required. Among all the methods belonging to this approach, we can quote those based on the mechanisms of natural selection, such as genetic algorithms and evolution strategies. ${ }^{30-33}$ One important advantage of these methods is the possibility to converge almost systematically toward a global minimum. However these methods are more time consuming in the sense that they require many evaluations of the objective function. To improve the computation time, more advanced methods have been proposed recently. For example, an evolution strategy method using the so-called meta-model (or response surface approximation) dramatically reduces the number of evaluations of the objective function. ${ }^{34}$ More details about this method are given in this article. Finally, hybrid methods are based on both methodologies. They generally consist of using first a global search algorithm with an evolutionary algorithm and then a local search algorithm, which allows it to converge faster toward the optimum value. The local search algorithm may be based on either the evaluation of the gradient of the objective function or on an approximation method.

In this paper, we present and validate an automatic and robust stochastic methodology to identify mechanical parameters in two different experimental configurations. A model data set has been obtained by modeling indentation tests with the finite element code ABAQUS. ${ }^{35}$ Mesh refinement, topography, finite element model, and convergence studies are also presented in the first part. The second part is devoted to the presentation of the inverse method and a description of the methodology used to identify the mechanical parameters with the best accuracy. We discuss in particular the evaluation of the starting parameters for the inverse method and the robustness of the solution. Two experimental situations have been treated. The first one corresponds to the identification of the mechanical parameters based on the experimental load-displacement curve and the residual imprint of the indented material obtained with only one conical indenter. The second one concerns the identification of the mechanical parameters with different load-displacement curves obtained with indenters of different conical semi-angles. As pointed out by one of the reviewers, this second approach, the dual indentation method, presupposes that the material properties are identical for the two locations tested by the two different indenter geometries. Many times, this is the case for large indentations where the penetration depths is in the micrometer-range; thus the volume tested is large compared to the grain size and the mechanical properties obtained representing average values for that material. In the case of nanoindentation experiments investigating the mechanical properties of the single component of advanced materials, where the penetration depth is as low as few tens of nanometers, the dual indentation method needs either an instrument capable of locating accurately the indentation position ${ }^{36}$ (same grain or phase) or a statistical study to discriminate any inhomogeneity in the mechanical properties. Indentation instruments with high position accuracy are, for example, in situ scanning electron microscopy (SEM) indentation or instruments capable of mapping the surface topography prior to indentation. We conclude by comparing the results of the inverse to the existing analytical approaches. We will also summarize the advantages and drawbacks of each configuration for this identification.

\section{FINITE ELEMENT MODEL}

Large deformation finite element (FE) indentation simulations were performed using the commercial $\mathrm{FE}$ code ABAQUS. For simplicity and to reduce computation time, the indentations were simulated using an axisymmetric model. Friction between the indenter and the indented body was neglected. 


\section{A. Mesh}

A total of 877 nodes and 808 quadrilateral elements have been used for the continuum, while the conical indenter was modeled as an analytical rigid body (see Fig. 2). To achieve a higher accuracy in the determination of the contact radius and the $z$-values of the residual imprint, the density of the mesh was optimized and refined close to the center of contact and gradually increased further away from the contact. The meshing strategy has been tested ${ }^{29}$ to be a best compromise between computing time and accuracy of the numerical results. Convergence studies and the insensitivity to the far-field effect from boundary conditions have also been carried out to ensure the validity of the results. Four different conical indenters have been tested whose semi-angles evolve between $\alpha=50^{\circ}$ and $80^{\circ}$ with an initial tip radius of $5 \mu \mathrm{m}$ (the most frequently used symbols are listed in Table I). The simulation was divided into three steps: contact, load, and unload.

\section{B. Constitutive law with an elastic-plastic hardening model}

A limitation of the inverse analysis is the lack of a generalized plastic constitutive law that can be used to characterize a broad number of metal materials. This limitation is shown by the number of different constitutive equations available in the literature $^{37}$ including

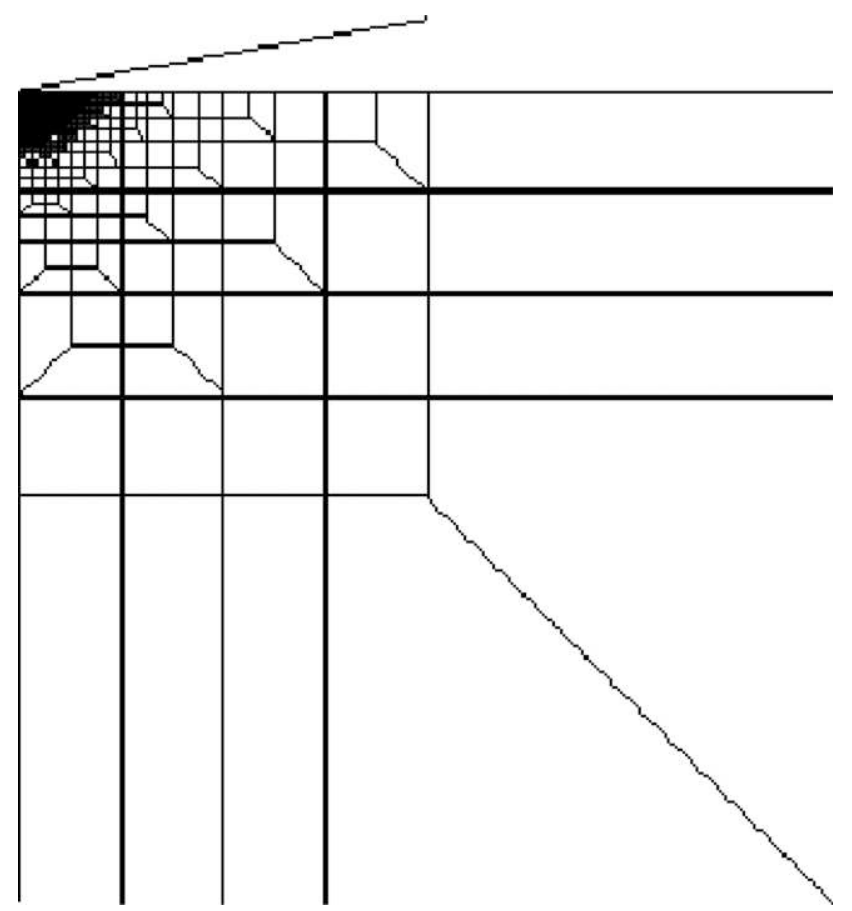

FIG. 2. Mesh structure to conduct the FE simulation of the indentation test with ABAQUS. To remove the effect of boundary conditions, infinite elements were used in a region far from the contact. Four nodes reduced integration axisymmetric elements $(\mathrm{C} 4 \mathrm{X} 4 \mathrm{R})$ are used in the contact zone.
Hollomon, ${ }^{38}$ Ludwik and Hollomon, ${ }^{39,40}$ Swift, ${ }^{41}$ and Ramberg and Osgood, ${ }^{42}$ to name a few.

The Ludwik-Hollomon law, a three-parameter constitutive law, has been implemented in this study to simulate the elastic-plastic behavior of the indented material. It has been found that, at least for many pure and alloyed metals, a fairly good approximation of the stress-strain behavior is represented by this work-hardening power law. ${ }^{39,40}$ Any other three-parameter constitutive law could be used by minimally varying the inverse method program code. The relationship between the total true stress $\sigma$ and the total true strain $\varepsilon$ is given by

$\sigma= \begin{cases}E \varepsilon & \text { for } \sigma \leq \sigma_{\mathrm{y}} \\ \sigma_{\mathrm{y}}\left(1+\frac{E}{\sigma_{\mathrm{y}}} \varepsilon_{\mathrm{P}}\right)^{n}=\sigma_{\mathrm{y}}\left(\frac{E}{\sigma_{\mathrm{y}}} \varepsilon\right)^{n} & \text { for } \sigma \geq \sigma_{\mathrm{y}}\end{cases}$

where $E$ is the Young's modulus of the material, $n$ is the strain hardening exponent, $\sigma_{\mathrm{y}}$ is the initial yield stress, and $\varepsilon_{\mathrm{p}}$ is the plastic strain:

$$
\left(\varepsilon_{\mathrm{p}}=\varepsilon-\frac{\sigma_{\mathrm{y}}}{E}\right)
$$

The von Mises criterion is assumed. Table II presents the theoretical mechanical parameters used for the simulations. We call them the target parameters $P_{\text {target }}$. These values have been chosen to represent a typical copper alloy. With the above assumption, the mechanical

TABLE I. List of symbols (most frequently used).

\begin{tabular}{|c|c|}
\hline \multicolumn{2}{|c|}{ List of symbols } \\
\hline$\alpha$ & Semi-angle of the conical indenter \\
\hline$E$ & Theoretical value of the Young's modulus \\
\hline$E^{i}$ & Value of the Young's modulus at $(\text { incr }=i)_{i=0, \ldots, f}$ \\
\hline$\varepsilon$ & Strain \\
\hline$N_{\text {obs }}$ & Number of observables \\
\hline$N_{\text {par }}$ & Number of parameters to identify \\
\hline $\mathrm{F}$ & Function to minimize \\
\hline$H$ & Current penetration depth \\
\hline Incr & Increment of the inverse method \\
\hline$L$ & Current load \\
\hline$P_{\text {target }}$ & $\begin{array}{l}\text { Theoretical values, target, of the parameters to describe the } \\
\text { mechanical behavior of the material }\end{array}$ \\
\hline$P$ & $\begin{array}{l}\text { Numerical parameters to describe the mechanical behavior of the } \\
\text { material }\end{array}$ \\
\hline$R_{\text {target }}$ & $\begin{array}{l}\text { Data set obtained obtained by the theoretical mechanical } \\
\text { properties using FE code ( } L-d \text { curve, topography...) }\end{array}$ \\
\hline$R$ & $\begin{array}{l}\text { Data obtained with the finite element model }(L-d \text { curve, } \\
\text { topography...) }\end{array}$ \\
\hline$N$ & Number of parameters to identify \\
\hline$N$ & Theoretical value of the strain hardening coefficient \\
\hline$n^{i}$ & Value of the strain hardening coefficient at $(\text { incr }=i)_{i=0, \ldots f}$ \\
\hline$\sigma$ & Stress \\
\hline$\sigma_{\mathrm{y}}$ & Theoretical value of the Yield stress \\
\hline$\sigma_{\mathrm{y}}^{i}$ & Yield stress at $(\text { incr }=i)_{i}=0, \ldots, f$ \\
\hline$W$ & Weight of the function to minimize \\
\hline
\end{tabular}


behavior of the material is fully determined with $E, \sigma_{\mathrm{y}}$, and $n$. The indenter is considered to be rigid since the diamond tip is extremely stiff $\left(E_{i}=1140 \mathrm{GPa}, v_{\mathrm{i}}=0.07\right)$. At the end of the simulation, the $L-h$ indentation curves and the topography of the corresponding residual imprint of the indented material are extracted using the FE code ABAQUS (see Fig. 3) for each conical indenter. The $L-h$ curve and the profile are plotted and will be called target curves $P_{\text {target }}$ as we will use these data to backfit the material properties.

In the following section, we present the inverse method and show how this method is used to identify the material parameters $P_{\text {target }}$ knowing the target data set $P_{\text {target }}$.

\section{INVERSE ANALYSIS METHODOLOGY}

For practical applications, the minimization method needs to ensure that the algorithm converges to the real solution with the required accuracy, whatever the initial set of parameters. The classical procedure is known as the least-square minimization problem, which approximates the computed data $R$ to the target data set data $R_{\text {target }}$ under variation of the material parameters $P$ :

$$
\text { Minimize } \chi(P)=\frac{1}{2}\left\|R_{\text {target }}-R(P)\right\|^{2} \text {. }
$$

To keep the parameters in a physically reasonable range, an upper $p_{j}^{\max }$ and a lower limit $p_{j}^{\min }$ need to be defined for each parameter. The index $j=\left(1, \ldots, N_{\mathrm{par}}\right)$, where $N_{\text {par }}$ is the number of parameters to identify. During the resolution of the minimization problem, the parameters are not allowed to exceed these limits.

Our approach has also been adapted to consider several $N_{\text {obs }}$ experimental data. A common difficulty with a

TABLE II. Theoretical mechanical properties $P_{\text {target }}$ of the indented material. Those mechanical properties are typical for copper alloys. In all simulations the Poisson's ratio $v$ is fixed at 0.3 .

\begin{tabular}{lcc}
\hline \hline$E(\mathrm{GPa})$ & $\sigma_{\mathrm{y}}(\mathrm{MPa})$ & $n$ \\
\hline 114 & 731 & 0.3 \\
\hline \hline
\end{tabular}

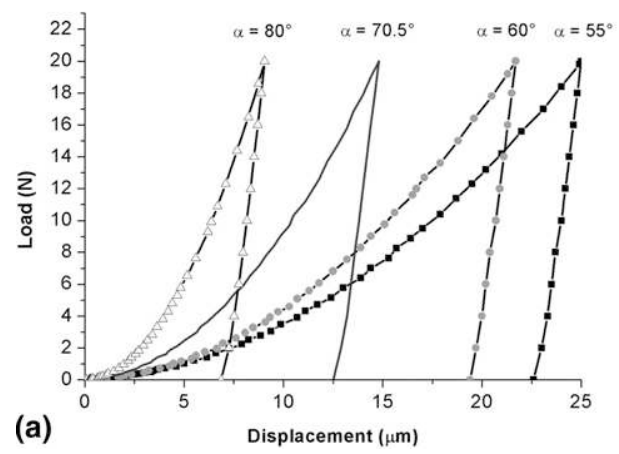

multiobjective function is the conflict between the objectives: in general, none of the feasible solutions is optimal at the same time. We have implemented the most classical one: the method of objective weighting for its efficiency and its ease of implementation. The multiobjective functions $\chi_{i}(P)$ are combined in one function $\mathrm{F}(P)$ such that:

$$
\left\{\begin{array}{c}
\mathrm{F}(P)=\sum_{i=1}^{N_{\mathrm{obs}}} w_{i} \chi_{i}(P) \\
\sum_{i=1}^{N_{\mathrm{obs}}} w_{i}=1, \text { with } 0 \leq w_{i} \leq 1
\end{array} .\right.
$$

A preference to one objective can be given by changing the corresponding weight $w$.

At each iteration incr of the inverse method, the communication between the FE simulation and the optimization algorithm was accomplished through a user interface written in the Python language. ${ }^{43}$ The module calls first a routine written in Fortran 77 initializing the different input files of the simulation and automatically launches the FE code. After each FE simulation, it calls a script to extract the numerical data of the simulation ( $L-h$ curve, topography, etc.). Eventually, the module calls a program coded in $C$ language to evaluate a new set of parameters $P$. This program can use two methods to generate a new set of parameters: a deterministic approach and a stochastic one based on Evolution Strategies. In the following, we present the Evolution Strategy method based on a meta-model.

\section{A. Evolution strategy}

Evolutionary algorithms (EA) are recognized now as robust and efficient optimization methods for a wide range of applications. These algorithms are based on the creation of a population of individuals, which correspond to the set of parameters that have to be identified. The evolution of this population toward the final result is based on the natural evolution phenomenon, through selection, recombination, and mutation rules. There are three main approaches: Genetic Algorithms (GA),

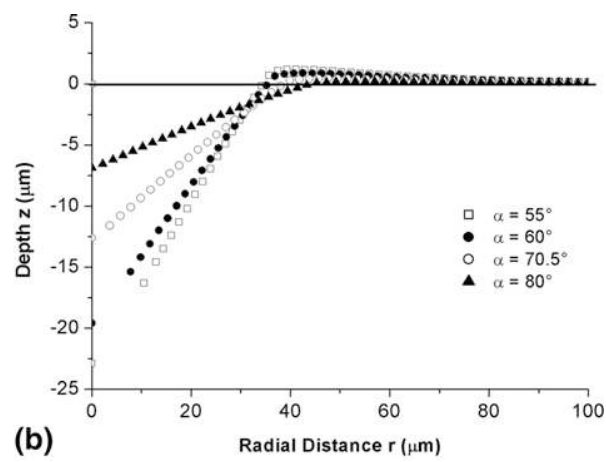

FIG. 3. Four different conical indenters are used for the indentations. The semi-angle $\alpha$ of the conical indenter varies between $55^{\circ}$ and $80^{\circ}$. (a) $L-h$ curve for each conical indenter. The maximum indentation load $L_{\max }$ is $20 \mathrm{~N}$. (b) Corresponding residual imprint after unloading. 
initially proposed by Holland in the sixties, ${ }^{44}$ which are based on a binary representation of the individuals; Evolution Strategies (ES), proposed initially by Rechenberg $^{32}$ and Schwefel, ${ }^{45}$ in which the individuals are represented by real values instead of binary ones. Both algorithms (GA and ES) are based on a Gaussian mutation operator. Evolutionary Programming $(\mathrm{EP})^{46}$ and Genetic Programming (GP) ${ }^{47}$ in which the evolution of the individuals is similar to the one of computer programs. ES are similar to GA, except that they use real coding parameters and that the selection of the parents is simpler. Mutation is also the main genetic operator, while recombination is not systematically used for producing new individuals. It is just the opposite for GA, which means that ES may find a solution faster, whereas GA are more robust to locate a global extremum.

In this paper, we use an enhanced version of ES based on a meta-model. ES operate on a population $\beta$ of individuals $I_{i}$. An individual (or chromosome) $I_{i}$ refers to a specific object parameter set $P_{i}$, its objective function value $F_{i}$, and a strategy parameter $s_{i}$. Index $i$ relates to the number of individuals generated within a population (it is fixed here at $\lambda=30$ ).

$$
I_{i}=\left(P_{i}, F_{i}, s_{i}\right) \quad .
$$

The algorithm is synthetized in Fig. 4. The initial population $\beta^{(0)}$ (generation $g=0$ ) is chosen randomly and uses the starting parameters of the inverse method. Consequently each decision parameter $p_{i=[1, \lambda], j=\left[1, N_{\mathrm{par}}\right]}^{0}$ of each individual $I_{i}^{0}$ of the initial population $\beta^{(0)}$ is found as follows:

$$
\left\{\begin{array}{c}
s_{i, j}^{0}=s \times \zeta_{i}\left(p_{j}^{\max }-p_{j}^{\min }\right) \\
p_{i, j}^{0}=p_{j}^{\min }+\zeta_{i}\left(p_{j}^{\max }-p_{j}^{\min }\right)
\end{array},\right.
$$

where $p_{j}^{\max }$ and $p_{j}^{\min }$ are the upper and lower limits of the parameter to identify and $s$ the starting parameter for the strategy parameter. $\zeta_{\mathrm{i}} \in[0,1]$ is a uniformly distributed random number generated for each value of $i$.

Within one ES generation step $g$, the inverse method evaluates the objective value $F_{i}$ for each set of parameters $P_{i}$ of the population $\beta$. Each generation $g$ then reproduces when a subset of individuals in the popula-

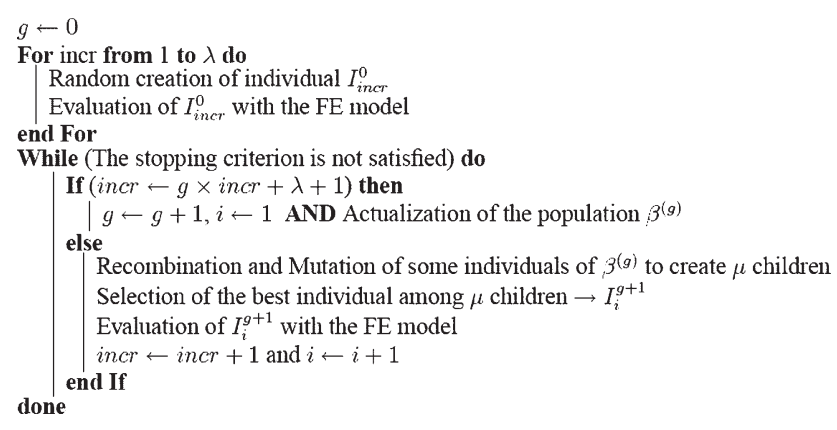

FIG. 4. Evolution Strategy in the inverse method. tion, called parents, are selected based on some specified criteria, and various search operators are applied to the parents to create an offspring (recombination, mutation). This offspring is evaluated with a fitness function $f$ and a selection is operated to progressively generate a new population $\beta^{(g+1)}{ }^{48}$ Finally, the termination condition is checked. For this, convergence criteria can be used or a maximum number of iterations incr can be fixed. Our algorithm is based on this second assumption. We describe here the different stages that are necessary to generate a new individual at each increment incr of the inverse method. Therefore we assume that the first generation $(g=0)$ has already been evaluated.

\section{Recombination}

Two main types of algorithms exist to make a child based on the previous generation $g$ : discrete or intermediary recombination. Our algorithm uses a global intermediary recombination, as the algorithm chooses randomly two parents $\left(P_{t, j}\right.$ and $P_{v, j}, t$ and $v$ are two random number $\in i=[1, \lambda])$ to generate a child:

$$
\left\{\begin{array}{l}
p_{i, j}^{g}=p_{t, j}^{g}+\zeta_{i}\left(p_{v, j}^{g}-p_{t, j}^{g}\right) \\
s_{i, j}^{g}=s_{i, j}^{g}+\zeta_{i}\left(s_{v, j}^{g}-s_{t, j}^{g}\right)
\end{array} .\right.
$$

This operation is realized $\mu$ times (we have fixed $\mu=7$ ). Typically, this stage ensures that offspring will have better fitness values than the parents; however, this is not guaranteed, due to the randomness inherent in the reproduction process. Even if $\left(p^{\prime}, s^{\prime}\right)$ does not survive during the "survivor selection" stage, our algorithm stores and includes these new individuals in the population. They are hence used as parents for the next increments of the inverse method which guarantees a large diversity among the individuals.

\section{Mutation}

The children $\left(p^{\prime}, s^{\prime}\right)$ are also mutated. Since the selection of the strategy parameter $s$ is highly dependent on the problem and its dimensionality, Schwefel ${ }^{45}$ proposed a concept referred to as self-adaptation, in which the mutation operator is applied to $p$ as well as $s$. This operation is realized by modifying dynamically $s_{i, j}^{g}$ and $P_{i, j}^{\prime g}$ according to

$$
\left\{\begin{array}{l}
p_{i, j}^{\prime g}=p_{i, j}^{\prime g}+N(0 ; 1) \cdot s_{i, j}^{g+1} \\
s_{i, j}^{g}=s_{i, j}^{g} \cdot e^{\Re(0 ; 1) \tau}
\end{array},\right.
$$

where $N(0 ; 1)$ denotes a vector of random Gaussian numbers with zero mean and one standard deviation. $\tau$ is called the "learning rate":

$$
\tau=\frac{1}{\sqrt{N_{\mathrm{par}}}} .
$$

The algorithm also has a boundary rule (Modified Interval Bounds Treatment). If the children parameter 
$p^{\prime}$ is outside the limits, its value is modified and set equal to the upper or lower limit of the interval $\left[p_{j}^{\min }, p_{j}^{\max }\right]$.

\section{Survivor selection}

After the generation of $\mu$ children, a selection is performed with the result of a new individual. At each increment of the inverse method incr, a method is used to choose the best child. This selection is based on the $(\mu, \lambda)$ version. One individual is selected, among the $\mu$ children, with the fitness function $f$. This fitness function measures how well an individual is adapted to the problem. This may be the same as the objective function of the problem being optimized, but often is not, in an effort to increase efficiency. Within survivor selection, we use the Kriging method to select one individual ${ }^{49}$ (and reject the others). We progressively generate a new population $\beta^{(g+1)}$ with $\lambda$ individuals.

The main advantages of ES are their easy implementation and the good quality of the approximate solution. They can treat simultaneously several individuals and are inherently parallel. Thus, the probability of reaching only a local minimum is reduced with respect to direct search methods. They are also more robust than direct search methods. Furthermore, the ES uses the information of the objective function, not derivatives or other auxiliary knowledge. It is thus well suited for problems in which the initialization is not intuitive and the cost functional may present several local minima. Consequently, this method is very well suited to identify the mechanical properties of the indented material. Meta-model-assisted evolution strategies ${ }^{34}$ help in reducing the number of evaluations of the objective function. Compared to classical ES, the main difference is that only $20 \%$ of the children population is exactly evaluated for each generation. The other $80 \%$ are evaluated using a high-dimensional interpolation model, called meta-model, which is dynamically built. It recycles the already calculated functions to better approximate the function over the optimization domain. For this, the Kriging method ${ }^{49}$ is used. This method provides both an estimation of the function value at any point but also the confidence interval of this estimation. The computation of the children using this approximate function is thus much faster.

\section{B. Validation of the algorithm}

We validate now the convergence of the algorithm. We use the FE model presented in Sec. II. The indenter has a semi-angle $\alpha$ equal to $80^{\circ}$. The yield stress $\sigma_{\mathrm{y}}$ and the strain hardening coefficient $n$ are fixed to the theoretical material properties shown in Table II.

The goal is to identify the Young's modulus $E$. We choose arbitrary a starting stiffness $E^{0}=147 \mathrm{GPa}$ (which corresponds to a $30 \%$ increase with respect to the theoretical material elastic modulus) and the search has been limited between 56 and $170 \mathrm{GPa}$. We compare here the target curves with the numerical $L-h$ curves.

After only 60 iterations, the inverse method converges to one solution: $E^{\mathrm{f}}=113.4 \mathrm{GPa}$. Compared to the theoretical material elastic modulus, it induces a relative error of $0.05 \%$ which permits to validate the efficiency of the evolution strategy for the following.

\section{Stability of the system}

We adopt a local approach to check the stability of the objective function using an example data set. We choose an initial set of values and simulate the indentation tests. The same computations are then repeated with a slight variation of parameters. The parameters are listed in Table II. We then make a variation of $1 \%$ on these parameters and evaluate the Gauss-Newton matrix $G$ :

$$
G=\sum_{k} \frac{\mathrm{d} h_{k}}{\mathrm{~d} P_{i}} \cdot \frac{\mathrm{d} h_{k}}{\mathrm{~d} P_{j}},
$$

where $h$ is the displacement reached during each simulation and $P$ is the set of parameters to identify. Therefore all values $h_{k}(P)$ are calculated using the first set of parameters, as well as perturbed values $h_{k}\left(P+\delta P_{i}\right)$, where $\delta P_{i}$ represents a small perturbation of the corresponding parameter $P$ :

$$
\frac{\mathrm{d} h}{\delta P_{i}}=\frac{h\left(P+\delta P_{i}\right)-h(P)}{\delta P_{i}} .
$$

The following symmetric matrix $G$ for the three parameters $\left(E, \sigma_{\mathrm{y}}, n\right)$ are obtained:

$$
G=\left(\begin{array}{ccc}
7.70 \times 10^{-9} & 1.20 \times 10^{-6} & 2.91 \times 10^{-3} \\
& 1.86 \times 10^{-4} & 0.45 \\
& & 1104.55
\end{array}\right) \text {. }
$$

First of all, we notice that this matrix is not well conditioned. After the computation of the eigenvalues we obtain a condition number equal to $\rho=6.96 \times 10^{12}$, which means that a small perturbation of the system may lead to a different global response of the material. The matrix $G$ can lead to the evaluation of the parameter correlation $C$ with the calculation of:

$$
C_{i j}=\cos \left(\frac{\mathrm{d} h}{\mathrm{~d} P_{i}}, \frac{\mathrm{d} h}{\mathrm{~d} P_{j}}\right)=\frac{G_{i j}}{\sqrt{G_{i i} G_{j j}}} .
$$

We obtain

$$
\begin{aligned}
& \cos \left(\frac{\mathrm{d} h}{\mathrm{~d} E}, \frac{\mathrm{d} h}{\mathrm{~d} \sigma_{y}}\right)=1, \\
& \cos \left(\frac{\mathrm{d} h}{\mathrm{~d} E}, \frac{\mathrm{d} h}{\mathrm{~d} h}\right)=0.9974, \\
& \cos \left(\frac{\mathrm{d} h}{\mathrm{~d} \sigma_{\mathrm{y}}}, \frac{\mathrm{d} h}{\mathrm{~d} h}\right)=0.9994,
\end{aligned}
$$


This reveals a strong correlation between the parameters to identify and proves the need for defining an adapted range for each material parameter before starting the identification. If the initial parameters are too far from the target values, the optimization process becomes lengthy. Furthermore, due to the large nonlinearity of the system and ill-conditioned nature of the problem treated here, it is probable that the desired solution, which corresponds to the global minimum of the function, cannot be reached.

To account for the instability of the system and the difficulties to converge to one unique solution, we suggest the following methodology: a preliminary study is done to determine the starting values of the inverse method through analytical models. Then multiple input data sets and numerical data sets are compared to ensure the robustness of the solutions. This hybrid approach of selecting the starting point from analytical models is comparable to regularization with the goal of focusing on a good spot and stabilizing the solution there. Two experimental situations have been evaluated. Method A uses the simulated target data given by one single indenter: the mechanical parameters are identified with the $L-h$ curve and the profile surface of the residual imprint after unloading. ${ }^{50}$ Method B uses two $L-h$ curves obtained with two conical indenters with different semi-angles. ${ }^{10}$

\section{METHOD A: DETERMINATION OF THE ELASTIC PLASTIC PROPERTIES WITH ONE SINGLE CONICAL INDENTER AND TOPOGRAPHY OF RESIDUAL IMPRINT}

We have chosen first to use a conical indenter with a semi angle equal to $70.5^{\circ}$. A distinctive feature of indentation experiments is that the material around the contact area tends to deform upward or downward around the vertical axis, where the load is applied. This behavior results in piling-up or sinking-in of material. The strain hardening exponent $n$ influences this surface deformation. $^{51-53}$ Therefore the strain hardening coefficient $n$ should be linked with the profile of the indented material after unloading. The coupling of the $L-h$ curve with the profile (Method A) is thus a technique to correlate these curves with the material properties.

\section{A. Preliminary study}

We demonstrate here the importance of the determination of the starting values to identify the mechanical parameters. The Young's modulus is fixed at its target value (see Table II). We consider arbitrary starting values for the plastic parameters: $\left(\sigma_{\mathrm{y}}^{0}=300 \mathrm{MPa}, n^{0}=0.05\right)$ far away from the theoretical material mechanical properties parameters used for the simulation. During the optimization procedure, these parameters can evolve between 290 and $1500 \mathrm{MPa}$ for $\sigma_{\mathrm{y}}$ and 0.01 and 0.53 for $n$. We suppose herein that the $L-h$ curve and the profile have the same impact on the calculated error $\mathrm{F}(P)\left(w_{1}=w_{2}=0.5\right)$.

Once the inverse analysis has converged, the following final results are obtained: $\sigma_{\mathrm{y}}^{f}=920.6 \mathrm{MPa}$ and $n^{f}=0.098$. It induces an error of $26 \%$ on $\sigma_{\mathrm{y}}$ and $67.3 \%$ on $n$. We consider here that the inverse method cannot identify the plastic parameters as the results are too far from the target values in Table II.

\section{B. Estimation of the starting parameters}

The Oliver and Pharr method ${ }^{5}$ has been widely adopted to determine the elastic properties of the indented material. It consists of fitting the unloading part of the $L-h$ curve to the power law relation derived from contact mechanics theory (see Fig. 5)

With the target $L-h$ curve (see Fig. 3), this method leads to the estimation of the reduced Young's modulus $E_{\mathrm{r}}=136 \mathrm{GPa}$, where:

$$
E_{\mathrm{r}}=\left(\frac{1-v^{2}}{E}+\frac{1-v_{i}^{2}}{E_{i}}\right)^{-1},
$$

and the Young's modulus: $E^{0}=143 \mathrm{GPa}$ which induces an error of $23 \%$ compared to the target value (Table II).

Other parameters to estimate are the yield stress $\sigma_{\mathrm{y}}$ and the strain hardening coefficient $n$. For elastic perfectly plastic materials, hardness $H$ is generally assumed to be three times the yield stress $\sigma_{\mathrm{y}}{ }^{3}$ Many models have

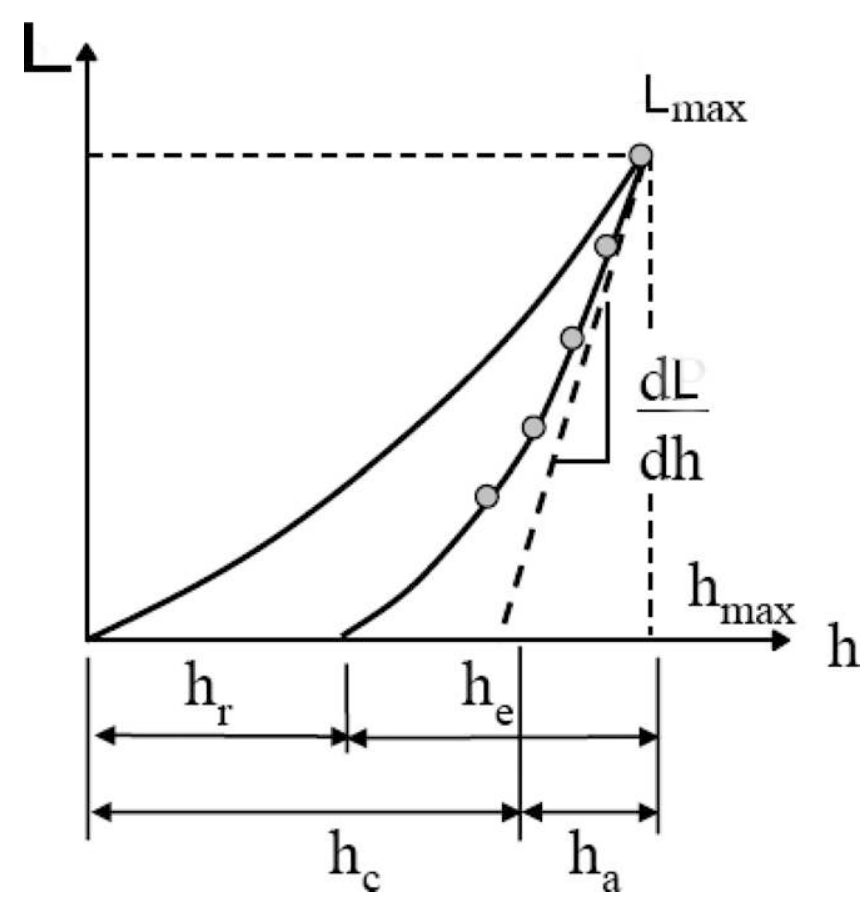

FIG. 5. Typical $L-h$ curve of an indentation test presenting the basic information that can be deduced for estimating material parameters. 
suggested this model be extended to elastic plastic materials with a strain hardening coefficient. ${ }^{40,54-56}$ However, these models are often dedicated to specific material behavior as elastic perfectly plastic behavior or reduced to elastic plastic behavior with a low strain hardening coefficient. The model introduced by Dao et al., ${ }^{12}$ which provides a unique solution from the $L-$ $h$ curve, is used in this study to estimate the starting parameters of the inverse method used. The drawback of this method concerns limiting conditions such as elastic-perfect plastic and perfect elastic materials. This method uses dimensional analysis to analyze the indentation response. During loading, $L$ is proportional to the square of the indenter displacement $h:{ }^{57-59}$

$$
L=C h^{2},
$$

where $C$ is the loading curvature. From the known parameters $C$ and $E_{\mathrm{r}}$, the dimensionless function $\Pi_{1}$ can be used to determine $\sigma_{0.033}$ :

$$
\begin{aligned}
\Pi_{1}= & \frac{C}{\sigma_{0.033}}=-1.131 \ln \left(\frac{E_{\mathrm{r}}}{\sigma_{0.033}}\right)^{3} \\
& +13.635 \ln \left(\frac{E_{\mathrm{r}}}{\sigma_{0.033}}\right)^{2} \\
& -30.594 \ln \left(\frac{E_{\mathrm{r}}}{\sigma_{0.033}}\right)+29.237
\end{aligned}
$$

This equation gives $\sigma_{0.033}=1580 \mathrm{MPa}$. The strain hardening coefficient $n$ is then determined by the dimensionless function $\Pi_{2}$.

$$
\begin{aligned}
\Pi_{2}=\frac{1}{E_{\mathrm{r}} h_{\max }} \frac{\mathrm{d} L}{\mathrm{~d} h} h_{h=h_{\max }} & C_{1} \ln \left(\frac{E_{\mathrm{r}}}{\sigma_{0.033}}\right)^{3} \\
& +C_{2} \ln \left(\frac{E_{\mathrm{r}}}{\sigma_{0.033}}\right)^{2} \\
& +C_{3} \ln \left(\frac{E_{\mathrm{r}}}{\sigma_{0.033}}\right)+C_{4}
\end{aligned}
$$

where $\left(C_{i}\right)_{i}=1.4$ are given by Table III. Table III This gives $n^{0}=0.123$. These two data lead to the estimation of the yield stress by using the Ludwik-Hollomon law:

TABLE III. Tabulated functions ${ }^{12}$ to determine $n$.

\begin{tabular}{ll}
\hline \hline$C_{1}$ & $-1.40557 n^{3}+0.77526 n^{2}+0.15830 n-0.06831$ \\
$C_{2}$ & $17.93006 n^{3}-9.22091 n^{2}-2.37733 n+0.86295$ \\
$C_{3}$ & $-79.99715 n^{3}+40.55620 n^{2}+9.00157 n-2.54543$ \\
$C_{4}$ & $122.65069 n^{3}-63.88418 n^{2}-9.58936 n+6.20045$
\end{tabular}

$$
\sigma_{0.033}=\sigma_{\mathrm{y}}\left(1+\frac{E^{0}}{\sigma_{\mathrm{y}}} 0.033\right)^{n} .
$$

We thus obtain $\sigma_{\mathrm{y}}^{0}=1060 \mathrm{MPa}$. Table IV summarizes the mechanical parameters obtained by the analytical solution. These results underline the possibility to approach $P_{\text {target }}$ but the measured relative error (compared to the target values) highlights the limited accuracy of the results. The reasons for such significant errors in the yield stress and strain hardening exponent values are linked to the high sensitivity of these parameters to the uncertainties in the indentation response parameters. ${ }^{23,24}$ We thus study how the inverse method can improve the identification of these parameters.

\section{Identification of the mechanical parameters}

We perform two studies. The first one corresponds to the identification of the two plastic parameters $\sigma_{\mathrm{y}}$ and $n$ by fixing the Young's modulus $E$ at its exact value. This approach is also used in practical applications as the Young's modulus is often known and the plastic properties of an alloy or a metal vary with thermal annealing or deformation hardening. The second study concerns the identification of elastic and plastic parameters. In each configuration we use the results of Sec. IV. B to fix the starting parameters at $P^{0}$ (see Table IV). During the optimization algorithm, $\sigma_{\mathrm{y}}$ (respectively $n$ ) can vary between 636 and $1484 \mathrm{MPa}$ (respectively 0.1 and 0.5 ). In the second configuration, the Young's modulus $E$ can vary between 100 and $186 \mathrm{GPa}$. These limits have been chosen based on the following idea. Usually, for metals, the mechanical properties of the indented material are generally known within an error of $\pm 30 \%$. Consequently, we consider here that they are sufficiently large to converge to the optimal set of parameters. In both cases the inverse method converges after 60 iterations. Table V presents the identified parameters in both configurations.

In the first study, the relative error shows that the identification gives more accurate results with the inverse

TABLE IV. Identified mechanical parameters $P^{0}$ obtained with the analytical method.

\begin{tabular}{ccc}
\hline \hline$E^{0}(\mathrm{GPa})$ & $\sigma_{\mathrm{y}}^{0}(\mathrm{MPa})$ & $n^{0}$ \\
\hline $143(+25 \%)$ & $1060(+44 \%)$ & $0.17(-43 \%)$ \\
\hline \hline
\end{tabular}

TABLE V. Identified mechanical parameters for Method A. Two studies have been performed. The first one corresponds to the identification of the plastic parameters (2). The second one corresponds to the identification of elastic and plastic parameters (3).

\begin{tabular}{cccc}
\hline \hline $\begin{array}{c}\text { Number of parameters } \\
\text { to identify }\end{array}$ & $E^{f}(\mathrm{GPa})$ & $\sigma_{\mathrm{y}}^{\mathrm{f}}(\mathrm{MPa})$ & $n^{f}$ \\
\hline $2\left(\sigma_{\mathrm{y}} ; n\right)$ & & $821(+12 \%)$ & $0.17(-43 \%)$ \\
$3\left(E, \sigma_{\mathrm{y}} ; n\right)$ & $112(+1.32 \%)$ & $864(+18 \%)$ & $0.23(-23 \%)$ \\
\hline \hline
\end{tabular}


method than with the analytical solution. Moreover, it proves the robustness of Method A. Although the chosen range of sets for each parameter to identify is large $( \pm 40 \%$ for $\sigma_{\mathrm{y}}$ and $\pm 70 \%$ for $n$ ), Method A leads to reasonable values. This result leads to the following question. What is the most important factor: the starting parameters or the imposed limits to obtain a good identification? In this way, we perform once again the first study by keeping the same range for each parameter but by arbitrarily modifying the starting values: $\sigma_{\mathrm{y}}^{0}=800 \mathrm{MPa}$ and $n^{0}=0.4$. The identified mechanical parameters are as follows: $\sigma_{\mathrm{y}}^{\mathrm{f}}=881 \mathrm{MPa}$ and $n^{f}=0.21$. The results are different than those obtained previously and the influence of the starting parameters. Moreover, it underlines the importance of upper and lower limits during the operation of the research algorithm. This conclusion is also confirmed by Eq. (5), where more importance is given to the limits than the starting parameters in the evaluation of a starting population $\beta^{(0)}$.

In the second study, we observe that the inverse method also converges well to $P_{\text {target }}$. This convergence is very accurate for the identification of the Young's modulus and more difficult for the evaluation of the plastic parameters. Whereas the Evolution Strategy avoids a local minimum of the cost function $\mathrm{F}(P)$, this result shows that the algorithm does not permit a convergence to the exact set of parameters $P_{\text {target }}$.

Method A leads to one solution whatever the starting parameters or the set of range in the case of "reasonable" starting values. These conclusions are highly correlated to the use of the Evolution Strategy. Hence, the conclusions of Stauss ${ }^{29}$ were different as the use of the deterministic approach induced the determination of well chosen starting values even by coupling the $L-h$ curve with the topography of the indented material. An advantage of Method A is that the experimental, or in this case the inputed target data set, measure of the profile is independent of the indentation response and consequently independent of external effects and machine compliance. From an experimental viewpoint, however, an accurate image of the profile can be time consuming and sometimes difficult to obtain.

\section{METHOD B: DETERMINATION OF THE ELASTIC PLASTIC PROPERTIES WITH SEVERAL CONICAL INDENTERS}

Instead of coupling the $L-h$ curves with the profile, we now identify the material parameters with two $L-h$ curves from two conical indenters with different semiangles. As an example, we consider here the dual indenter geometry of a $60^{\circ}$ and $80^{\circ}$ pair. The main drawback of this method compared to method A is the computation time as for each inverse analysis iteration incr, two FE simulations are performed. On the other hand, the measurement of a second L-h curve may be less time consuming then measurement of the residual indent topography, in particular for micro and microscopic indents.

\section{A. Estimation of the starting parameters with the method developed by Bucaille et al.}

We use the same methodology as in Sec. IV. B to estimate the starting parameters and evaluate the Young's modulus with each $L-h$ obtained for each conical indenter. An average of these values gives us an initial Young's modulus equal to $E^{0}=139 \mathrm{GPa}$.

Using the different target $L-h$ curves (see Fig. 3), Bucaille et al. ${ }^{10}$ and later Chollacoop et al. ${ }^{11}$ extended the method proposed by Dao et al. ${ }^{12}$ for forward and reverse analysis based on dual sharp indenters. As for the Dao et al. method, the drawback of these methods is the problem with asymptotic analysis regarding limiting conditions such as elastic-perfect plastic and perfect elastic materials. This method consists of using dimensionless functions to rebuild the $\sigma-\varepsilon$ curve based on several points $\left(\varepsilon_{\mathrm{r}} ; \sigma_{\mathrm{r}}\right)$, from load-displacement curves of different conical indenters. Chollacoop et al., ${ }^{11}$ Dao et al., ${ }^{12}$ Tho et al., ${ }^{60}$ and Zeng and $\mathrm{Chiu}^{33}$ have demonstrated the robustness of the solution when the method is based on dual sharp indenters. This method has been used to assess stress strain properties of thin films ${ }^{61,62}$ and the properties of the matrix of metal matrix composites. ${ }^{36}$

In conical indentation, strain increases continuously with the indentation depth $h$, and an appropriate equivalent expression is

$$
\varepsilon_{\mathrm{r}}=0.105 \cot (\alpha)
$$

where $\varepsilon_{\mathrm{r}}$ is the strain accumulated beyond the yield point. Bucaille et al. ${ }^{10}$ have proposed a set of universal dimensionless functions to link the stress $\sigma_{\mathrm{r} \alpha}$ value in one point of the $\sigma-\varepsilon$ curve of the indented material with the reduced Young's modulus of the system $E_{\mathrm{r}}$ and the semi-angle $\alpha$ of the conical tip.

$$
\begin{aligned}
\Pi_{1 \alpha}= & \frac{C_{\alpha}}{\sigma_{\mathrm{r} \alpha}}=0.02552 \tan ^{2} \theta\left[\ln \left(\frac{E_{\mathrm{r}}}{\sigma_{\mathrm{r} \alpha}}\right)\right]^{3} \\
& -0.72526\left[\ln \left(\frac{E_{\mathrm{r}}}{\sigma_{\mathrm{r} \alpha}}\right)\right]^{2} \\
& +6.34493\left[\ln \left(\frac{E_{\mathrm{r}}}{\sigma_{\mathrm{r} \alpha}}\right)\right]-6.474458
\end{aligned}
$$

Table VI summarizes the analytical results obtained and the corresponding relative error. It shows that the Bucaille method gives better results than the classical analytical method with only one conical indenter. 


\section{B. Identification of the plastic parameters}

We fix the Young's modulus $E$ at its exact value and we identify the plastic parameters $\sigma_{\mathrm{y}}$ and $n$ using Table VI to fix the starting plastic parameters. Table VII presents the final results $P^{f}$ obtained. We observe here an improvement of the method compared to the Bucaille one described above.

The yield stress $\sigma_{\mathrm{y}}^{f}$ obtained is better than those identified by the Bucaille method as the relative error decreases from $15 \%$ for the Bucaille method to $8 \%$ for the inverse method and the same phenomenon is observed for $n$. We now test the accuracy of the method when three parameters have to be identified.

\section{Identification of the elastic plastic parameters}

We consider here that no parameters are known. We have hence to identify the elastic and the plastic parameters. If the number of parameters increases, the efficiency and the convergence of the optimization technique cannot be guaranteed. Consequently, the process is the following: we identify first the Young's modulus $E^{f}$ by fixing the initial yield stress $\sigma_{\mathrm{y}}$ and the strain hardening exponent $n$ to the value identified in Sec. V. A (see Table VI). Once the elastic parameter has been identified, we will identify the plastic

TABLE VI. Mechanical parameters $P^{0}$ identified with the Bucaille method.

\begin{tabular}{lcc}
\hline \hline$E^{0}(\mathrm{GPa})$ & $\sigma_{\mathrm{y}}^{0}(\mathrm{MPa})$ & $n^{0}$ \\
\hline $139(+23 \%)$ & $580(-20 \%)$ & $0.35(+17 \%)$ \\
\hline \hline
\end{tabular}

TABLE VII. Identification of the plastic parameters with Method B. The Young's modulus $E$ is fixed here at its experimental value $(E=114 \mathrm{GPa})$.

\begin{tabular}{lc}
\hline \hline$\sigma_{\mathrm{y}}^{f}(\mathrm{MPa})$ & $n^{f}$ \\
\hline $676(-8 \%)$ & $0.33(+11 \%)$ \\
\hline \hline
\end{tabular}

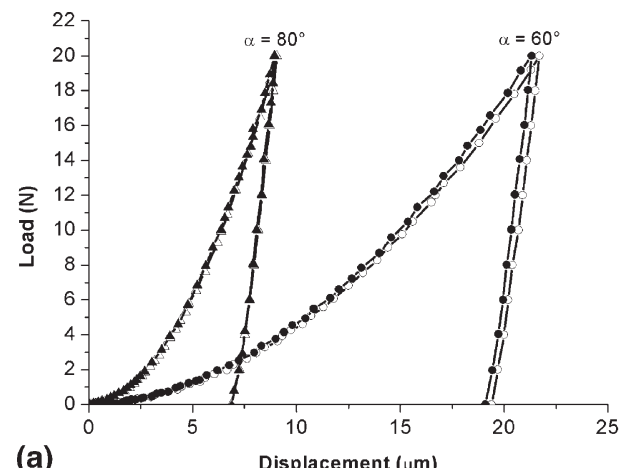

(a) parameters $\sigma_{\mathrm{y}}$ and $n$ by fixing the identified Young's modulus $E^{f}$.

We use Sec. V. A to define the starting Young's modulus at $E^{0}=139 \mathrm{GPa}$. This value can vary at $\pm 30 \%$ during the inverse method. After $6 \mathrm{~h}$ of calculations on a state-of-the-art personal computer, the inverse method converges easily to a unique value: $E^{f}=122 \mathrm{GPa}$. This means a relative error of almost $8 \%$ (compared to the target value), which is three times lower than for the Bucaille method. We also suppose that the inverse method would be more accurate if the fixed yield stress and the strain hardening coefficient $n$ would be the same as the theoretical ones.

$E$ is now fixed at $122 \mathrm{GPa}$ and the plastic parameters are now identified. Table VI presents the starting values $P^{0}$ used for the plastic parameters. Each plastic parameter can vary $\pm 30 \%$ during the inverse method. Table VIII presents the results obtained for different weight function $w_{i}$. If $w_{1}=w_{2}=0.5$, the results obtained with two conical indenters have the same influence on the identification process. If $w_{1}>w_{2}$ (respectively $w_{1}<$ $w_{2}$ ), and the results obtained with the sharper (respectively the larger) indenter have more influence than the larger (respectively the sharper) one. Table VIII shows that the inverse method has a better accuracy than the Bucaille method. It underlines also that the weights have less influence on the parameters. Figure 6 presents a comparison between $R_{\text {target }}$ and $R$. We observe a very good accuracy between each $L-h$ curves.

TABLE VIII. Plastic parameters identified with Method B. $E$ is fixed at $122 \mathrm{GPa}$. The weight functions vary to evaluate the impact of each curve on the accuracy of the identification. $w_{1}$ is the weight associated to the $60^{\circ}$ conical semi-angle and $w_{2}$ is the weight associated to the $80^{\circ}$ conical semi-angle.

\begin{tabular}{ccc}
\hline \hline \multicolumn{1}{c}{ Weights } & \multicolumn{1}{c}{$\sigma_{\mathrm{y}}^{f}(\mathrm{MPa})$} & \multicolumn{1}{c}{$n^{f}$} \\
\hline$w_{1}=w_{2}=0.5$ & $661(-9 \%)$ & $0.33(+9 \%)$ \\
$w_{1}=0.75 ; w_{2}=0.25$ & $644(-12 \%)$ & $0.34(+13 \%)$ \\
$w_{1}=0.25 ; w_{2}=0.75$ & $660(-10 \%)$ & $0.33(+9 \%)$ \\
\hline \hline
\end{tabular}

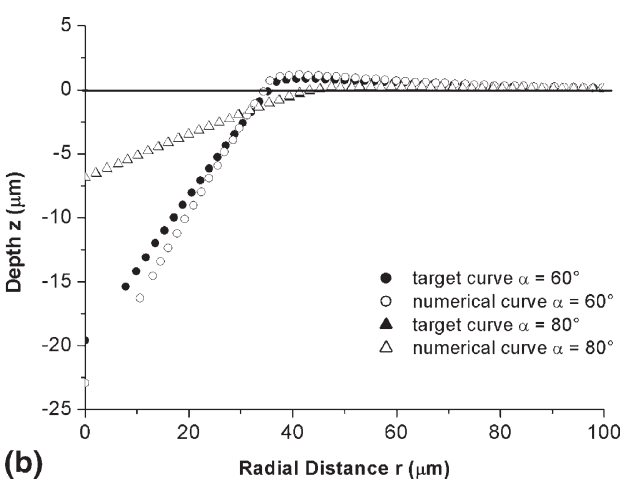

FIG. 6. Comparison between target (solid symbols) and numerical (empty symbols) curves in case of Method B. We have used the mechanical parameters obtained when $w_{1}=w_{2}=0.5$ : (a) $L-h$ curves and (b) residual imprints after unloading. 


\section{CONCLUSIONS}

In this study, we propose a new approach that can be used to identify elastic plastic material parameters using indentation tests. This method is based on the coupling of a semi analytical solution with an Evolution Strategy Algorithm through an inverse analysis. This coupling is necessary in order for the inverse method to improve the accuracy of the solution since the indentation process is highly nonlinear. The proposed model has shown its robustness and can be considered an efficient tool to determine material properties.

Our approach is divided in two steps. First a semi-analytical study leads to the estimation of starting parameters. Then the inverse method enables the identification of the elastic and plastic parameters with accuracy. Theoretical verification is performed on two simulated configurations. Method A is based on the $L-h$ curve and the residual imprint of the material after unloading, indented with one conical indenter. Method B is based on the $L-h$ curves of a material indented with different conical indenters. Both methods achieve reasonable accuracy. Whereas the computation time is higher in case of Method B, its accuracy is greater than the one of Method A. This better response is mainly due to a good estimation of the starting parameters (and consequently boundary limits), which can be obtained by introducing some physical knowledge, such as using the method developed by Bucaille et al., ${ }^{10}$ in solving the indentation inverse problem.

\section{ACKNOWLEDGMENTS}

Financial support by the Competence Center Energy and Mobility through project "Platform for High Temperature Materials" is gratefully acknowledged. Special Thanks go to S. Stauss, EMPA, for the permission to use his FE mesh.

\section{REFERENCES}

1. J.L. Loubet, J.M. Georges, O. Marchesini, and G. Meille: Vickers indentation curves of magnesium-oxide $(\mathrm{MgO})$. J. Tribol. 106, 43 (1984).

2. J.B. Pethica, R. Hutchings, and W.C. Oliver: Hardness measurement at penetration depths as small as $20 \mathrm{~nm}$. Philos. Mag. A 48, 593 (1983).

3. S. Stauss, P. Schwaller, J-L. Bucaille, R. Rabe, L. Rohr, J. Michler, and E. Blank: Determining the stress-strain behaviour of small devices by nanoindentation in combination with inverse methods. Microelectron. Eng. 67-68, 818 (2003).

4. M.F. Doerner and W.D. Nix: A method for interpreting the data from depth-sensing indentation. J. Mater. Res. 1, 601 (1986).

5. W.C. Oliver and G.M. Pharr: An improved technique for determining hardness and elastic modulus using load and displacement sensing indentation experiments. J. Mater. Res. 7, 1564 (1992).

6. D. Tabor: The Hardness of Metals (Clarendon Press, Oxford, UK, 1951).
7. Y.T. Cheng and C.M. Cheng: Scaling approach to conical indentation in elastic-plastic solids with work hardening. J. Appl. Phys. 84, 1284 (1998).

8. Y.T. Cheng and C.M. Cheng: Scaling, dimensional analysis, and indentation measurements. Mater. Sci. Eng., R 44, 91 (2004).

9. K.D. Bouzakis, N. Michailidis, and G. Erkens: Thin hard coatings stress-strain curve determination through a FEM supported evaluation of nanoindentation test results. Surf. Coat. Technol. 142, $102(2001)$

10. J.L. Bucaille, S. Stauss, E. Felder, and J. Michler: Determination of plastic properties of metals by instrumented indentation using different sharp indenters. Acta Mater. 51, 1663 (2003).

11. N. Chollacoop, M. Dao, and S. Suresh: Depth-sensing instrumented indentation with dual sharp indenters. Acta Mater. 51, 3713 (2003).

12. M. Dao, N. Chollacoop, K.J. Van Vliet, T.A. Venkatesh, and S. Suresh: Computational modeling of the forward and reverse problems in instrumented sharp indentation. Acta Mater. 49, 3899 (2001).

13. A.E. Giannakopoulos and S. Suresh: Determination of elastoplastic properties by instrumented sharp indentation. Scr. Mater. 40 , 1191 (1999).

14. N. Huber and C. Tsakmakis: Determination of constitutive properties from spherical indentation data using neural networks. Part I: The case of pure kinematic hardening in plasticity laws. J. Mech. Phys. Solids 47, 1569 (1999).

15. B. Backes, K. Durst, and M. Goken: Determination of plastic properties of polycrystalline metallic materials by nanoindentation: Experiments and finite element simulations. Philos. Mag. 86, 5541 (2006)

16. Y.P. Cao and N. Huber: Further investigation on the definition of the representative strain in conical indentation. J. Mater. Res. 21, 1810 (2006).

17. I.S. Choi, M. Dao, and S. Suresh: Mechanics of indentation of plastically graded materials. I: Analysis. J. Mech. Phys. Solids 56, 157 (2008).

18. B. Guelorget, M. Francois, C. Liu, and J. Lu: Extracting the plastic properties of metal materials from microindentation tests: Experimental comparison of recently published methods. J. Mater. Res. 22, 1512 (2007).

19. X.Q. Qian, Y.P. Cao, and J. Lu: Dependence of the representative strain on the hardening functions of metallic materials in indentation. Scr. Mater. 57, 57 (2007).

20. S. Swaddiwudhipong, E. Harsono, J. Hua, and Z.S. Liu: Reverse analysis via efficient artificial neural networks based on simulated Berkovich indentation considering effects of friction. Eng. Comput. 24, 127 (2008).

21. T.W. Capehart and Y.T. Cheng: Determining constitutive models from conical indentation: Sensitivity analysis. J. Mater. Res. 18, 827 (2003)

22. A. Gouldstone, N. Chollacoop, M. Dao, J. Li, A.M. Minor, and Y.L. Shen: Indentation across size scales and disciplines: Recent developments in experimentation and modeling. Acta Mater. 55, 4015 (2007)

23. H. Lan and T.A. Venkatesh: Determination of the elastic and plastic properties of materials through instrumented indentation with reduced sensitivity. Acta Mater. 55, 2025 (2007).

24. H. Lan and T.A. Venkatesh: On the uniqueness and sensitivity issues in determining the elastic and plastic properties of powerlaw hardening materials through sharp and spherical indentation. Philos. Mag. 87, 4671 (2007).

25. J.L. Cohon: MultiObjective Programming and Planning (Academic Press, New York, 1978).

26. J.C. Gilbert: Differentiable Optimization: Theory and Algorithms (INRIA, Roquencourt, France, 1999). 
27. P. Pilvin: Multiscale approach for the prediction of the materials andastic component, Ph.D. Thesis, Universite de Paris VI, France (1983).

28. M. Bocciarelli and G. Maier: Indentation and imprint mapping method for indentation of residual stresses. Comp. Mater. Sci. 39, 381 (2007).

29. S.N. Stauss-Ueno: Assessment of mechanical properties using instrumented indentation and inverse methods, Ph.D. Thesis, EPF Lausanne, Switzerland, (2006).

30. D.E. Goldberg: Genetic Algorithms in Search, Optimization and Machine Learning (Addison-Wesley, Reading, MA, 1989).

31. Z. Michalewicz: Genetic Algorithms + Data Structures $=$ Evolution Programs (Springer-Verlag, Berlin, Germany, 1992).

32. I. Rechenberg: Evolutionsstrategie: Technical System Optimization from Biological Evolution Principle (Frommann-Holzboog, Stuttgart, Germany, 1973).

33. K. Zeng and C.H. Chiu: An analysis of laod-penetration curves from instrumented indentation. Acta Mater. 49, 3539 (2001).

34. M. Emmerich, A. Giotis, M. Özdemir, T. Bäck, and K. Giannakoglou: Metamodel-assisted evolution strategies, in Parallel Problem Solving from Nature VII, edited by J.J. Merelo Guervss, P. Adamidis, H.G. Bever, J-L. Fernández-Villacañas, and H-P. Schwefel (Springer, Secaucus, NJ, 2002).

35. ABAQUS—Standard User's manual version 6.4 (Hibbit, Karlsson and Sorensen Inc., 2002)

36. J.L. Bucaille, A. Rossoll, B. Moser, S. Stauss, and J. Michler: Determination of the matrix in situ flow stress of a continuous fibre reinforced metal matrix composite using instrumented indentation. Mater. Sci. Eng., A 369, 82 (2004).

37. Z. Gronostajski: The constitutive equations for FEM analysis. J. Mater. Process. Technol. 106, 40 (2000).

38. J.H. Hollomon: Tensile deformation. Trans. Am. Inst. Mining Metall. Eng. 162, 268 (1945).

39. G. Dieter: Mechanical Metallurgy, 2nd ed. (Mc Graw-Hill, New York, 1976).

40. J. Lubliner: Plasticity Theory (Macmillan, New York, 1990).

41. H.W. Swift: Plastic instability under plane stress. J. Mech. Phys. Solids 1, 1 (1952).

42. W. Ramberg and W.R. Osgood: Description of stress-strain curves by three parameters. National Advisory Committee for Aeronautics, Technical Note 902 (1943).

43. Python Software Foundation (www.python.org, 2005).

44. J.H. Holland: Outline for a logical theory of adaptive systems. J. Assoc. Computing Machinery 9, 297 (1962).

45. H-P. Schwefel: Numerical Optimization of Computer Models, 2nd ed. (JohnWiley and Sons, New York, 1995).

46. L.J. Fogel: On the organization of intellect, Ph.D. Dissertation, UCLA, CA, (1964)
47. N. Cramer: A representation for the adaptive generation of simple sequential programs, in Genetic Algorithms and the Applications, edited by J. Grefenstette (Lawrence Erlbaum Associates, Mahwah, NJ, 1985), p. 183.

48. T. Bäck, D. Fogel, and Z. Michalewicz: Handbook of Evolutionary Computation (Oxford University Press, Oxford, UK, 1997).

49. S. Sakata, F. Ashida, and M. Zako: Structural optimization using Kriging approximation. Comput. Methods Appl. Mech. Eng. 192 923 (2003)

50. O. Ghouati and J.C. Gelin: Gradient based methods, genetic algorithms and the finite element method for the identification of material parameters, in Simulation of Materials Processing: Theory, Methods and Applications, edited by J. Huétink and F.P.T. Baaijens (A.A. Balkema, Rotterdam, The Netherlands, 1998), p. 157.

51. J. Alcalá, A.C. Barone, and M. Anglada: The influence of plastic hardening on surface deformation modes around Vickers and spherical indents. Acta Mater. 48, 3451 (2000).

52. M.M. Chaudhri and M. Winter: The load-bearing area of a hardness indentation. J. Phys. D 21, 370 (1988).

53. R. Hill, B. Storakers, and A. Zdunek: A theoretical study of the Brinell hardness test. Proc. R. Soc. London A 423, 301 (1989).

54. X.Y. Feng and T.C. Wang: Scaling functions in conical indentation of elastic-plastic solids. Acta Mech. 196, 245 (2008).

55. X-L. Gao, X.N. Jing, and G. Subhash: Two new expanding cavity models for indentation deformations of elastic strain-hardening materials. Int. J. Solids Struct. 43, 2193 (2006)

56. W. Zielinski, H. Huang, and W.W. Gerberich: Microscopy and microindentation mechanics of single crystal Fe-3 wt.\% Si: Part II. TEM of the indentation plastic zone. J. Mater. Res. 8, 1300 (1993).

57. J.W. Harding and I.N. Sneddon: The elastic stresses produced by the indentation of the plane surface of a semi-infinite elastic solid by a rigid punch. Proc. Camb. Philol. Soc. 41, 16 (1945).

58. A.E.H. Love: Boussinesq's problem for a rigid cone. Q. J. Math. 10, 161 (1939).

59. I.N. Sneddon: The relation between load and penetration in the axisymmetric Bossinesq problem for a punch of arbitrary profile. Int. J. Eng. Sci. 3, 47 (1965).

60. K.K. Tho, S. Swaddiwudhipong, Z.S. Liu, K. Zheng, and J. Hua: Uniqueness of reverse analysis from conical indentation tests. J. Mater. Res. 19, 2498 (2004).

61. J.L. Bucaille, S. Stauss, P. Schwaller, and J. Michler: A new technique to determine the elastic properties of thin metallic films using sharp indenters. Thin Solid Films 447-448, 239 (2004).

62. S. Swaddiwudhipong, K.K. Tho, Z.S. Liu, and K. Zeng: Material characterization based on dual indenters. Int. J. Solids Struct. 42, 69 (2005). 